tin repression. It seems that HP1a propagation occurs independently of local chromatin organization defined by regulatory elements.

This work is supported by RFBR (grants 17-04-01984, 17-00-00282) and RSCF 19-74-20178

References 1. Abramov, Y.A., et al., The Differences Between Cis- and Trans-Gene Inactivation Caused by Heterochromatin in Drosophila. Genetics, 2016. 202(1): p. 93-106.

doi: http://dx.doi.org/10.7124/bc.0009FE

\section{S-10. DNA repair toolbox - cellular ability to repair multiple simultaneously induced single-strand DNA breaks}

\author{
Oskar Szelest, Damian Kurleto, Jurek \\ W. Dobrucki \\ Department of Cell Biophysics, Faculty of Bio- \\ chemistry, Biophysics and Biotechnology, Jagiello- \\ nian University, Krakow \\ szelest.oskar@gmail.com
}

Cellular capacity to repair DNA damage is limited, partly due to a fact that the number of copies of nuclear proteins available for a task of DNA repair is limited. Two key players involved in repair of SSBs, XRCC1 (X-ray Repair Cross Complementing Protein 1), which is involved in short patch and long path repair of singlestrand DNA breaks (SSBs), and PCNA (Proliferating Cell Nuclear Antigen), which is primarily involved in DNA replication, are recruited to the site of damage. Aims. This research is focused on understanding the process of saturation of a capacity to repair single-strand DNA breaks, by means of imaging recruitment of XRCC1 and PCNA to DNA lesions induced in non-replicating and replicating cells. The goal of this work was to assess cellular capacity to recruit detectable numbers of PCNA and XRCC1 molecules to several DNA lesions induced in close succession or separated by time interval, and quantitate a process of saturation of repair capacity as a function of the number of damage sites and the position of a cell in the cell cycle. Methods. Local DNA damage (SSBs) was induced by exposing a small region of the cell nucleus to a focused beam of laser light 1 . Live cells, expressing GFP-PCNA and RFP-XRCC1, or cells stained by immunofluorescence, were imaged using confocal fluorescence microscopy. Results. XRCC1 was recruited to the induced DNA lesions in all phases of the cell cycle, in contrast to PCNA which was not recruited in early S phase. Recruitment of PCNA was detected exclusively in middle and late S-phase. When DNA SSBs were induced at short time intervals (seconds) in several locations (from 1 to 30 ) in the cell nucleus, RFP-XRCC1 was recruited to only 5-6 of them. The recruitment of GFP-PCNA was limited to an even lower number of damage spots. When DNA lesions were induced in close succession, the amount of XRCC1 was the highest in the first spot, and lower in each subsequent location, suggesting that the cell activated recruitment very quickly, and was significantly exhausting the stock of the repair protein by recruiting it to each subsequent DNA lesion. Interestingly, a small amount of XRCC1 and PCNA which was not recruited to any damage site always remained in the cell nucleus outside of the damaged regions. Immunofluorescence studies confirmed the results obtained with live cells expressing fusion proteins. Conclusions. Cellular ability to repair single-strand DNA breaks that were generated 
in different locations, in succession of each other, is limited to a just few SSBs spots. The cell appears to recruit very large number of copies of XRCC1 and PCNA to the first detected lesion rather than spread the resources over all the lesions. In early and late S-phase, when the number of damage sites exceeds approximately 3 , the available PCNA pool appears to be exhausted, while recruitment of XRCC1 is still detectable. This suggests switching repair mechanisms from long to short patch.

Project funded by the National Science Centre, Poland, grant no. 2017/27/N/NZ1/00975

References: 1. Solarczyk, K.J. et al. Inducing local DNA damage by visible light to study chromatin repair. DNA Repair (Amst). 11, 996-1002 (2012).

doi: http://dx.doi.org/10.7124/bc.0009FF

\section{T-1. Analysis of nuclear actin in human progeria cells.}

Yuto Takahashi ${ }^{1}$, Nanako Machida ${ }^{1}$, Tom Misteli $^{2}$, Robert Grosse ${ }^{3}$, Kei Miyamoto ${ }^{4}$, Masahiko Harata ${ }^{1}$.

\footnotetext{
${ }^{1}$ Graduated School of Agricultural Science, Tohoku University, Japan; ${ }^{2}$ National Cancer Institute, National Institutes of Health, USA; ${ }^{3}$ Institute of Pharmacology, BPC University of Marburg, Germany; ${ }^{4}$ Faculty of Biology-Oriented Science and Technology, Kinki University, Japan.

tear403forbidden@gmail.com
}

Hutchinson-Gilford progeria syndrome (HGPS) is a rare premature disorder caused by de novo single base substitution in the lamin A gene. Lamin A contributes to nuclear architecture and spatial organization of chromatin in the nucleus. The lamin A mutant in HGPS cells is called progerin. The expression of progerin leads to functional and structural disruption of nuclear organization. In HGPS cells, several nuclear defects are observed such as irregular nuclear shape and increased DNA damage. By in vitro assay, it was shown that lamin A directly interacts with actin in the nucleus [1]. Actin is abundantly present in cytoplasm and is involved in multiple cellular functions. In addition, some actin exists in the nucleus as monomeric globular (G-) actin and polymerized filamentous (F-) actin. Nuclear actin has roles in epigenetic regulation. For instance, nuclear F-actin is involved in transcriptional regulation, DNA damage repair, and activation of Wnt/beta-catenin signaling [2]. Since progerin lacks actin binding sites [1], we hypothesized that the expression of progerin impairs functions and dynamics of nuclear actin, and that these nuclear actin dysfunctions are relevant to HGPS phenotypes. Thus we analyzed nuclear actin in HGPS cells. In this research, we analyzed human dermal fibroblast cells, which inducibly express GFPprogerin by doxycycline (Dox) as HGPS model cells [3]. When we observed nuclear F-actin, we found that the HGPS cells contains less nuclear F-actin as compared to control cells, in which progerin expression is not induced. Consistent with this observation, the activity of a Wnt/beta-catenin targeting promoter is reduced in the HGPS cells. Next, to test the possibility that the reduction of nuclear F-actin is relevant to HGPS phenotypes, we artificially increased nuclear F-actin by expressing NLS (nuclear localization signal)-tagged actin in the HGPS cells. It was observed that the irregular nuclear shapes of the HGPS cells were complemented by the expression of NLS-actin. These results suggest that reduction of nuclear 